\title{
Reflection waveform inversion in acoustic VTI media
}

Yuanyuan Li and Tariq Alkhalifah, King Abdullah University of Science and Technology

\section{Summary}

Full waveform inversion (FWI) in transversely isotropic media with vertical symmetry axis (VTI) provides the opportunity to better match the data at the near and far offsets. However, multi-parameter FWI in general suffers from a serious cycle-skipping and trade-off problem. Reflection waveform inversion (RWI) can help us build a background model by minimizing the reflection data residuals. Thus, we apply RWI to acoustic VTI media. According to the radiation patterns analysis, the acoustic VTI media should be described by a combination of the normal-moveout (NMO) velocity $v_{n}$ and the anisotropic parameters $\eta$ and $\delta$ in the RWI applications. To reduce the trade-off, we first invert for the background $v_{n}$, and then update the background $v_{n}$ and $\eta$, simultaneously to fit the far-offset reflections. We apply Born modeling to produce the reflections for the two stages of the RWI method. For a follow up FWI applications, we use the background $v_{n}$ and $\eta$ to calculate the horizontal velocity $v_{h}$ and the parameters $\eta$ and $\varepsilon$. The acoustic VTI FWI will utilize the diving waves to improve the background, as well as utilize the reflections for high resolution information. We test the inversion algorithm on the modified VTI Sigsbee 2A model (a salt free part). The results show that the approach can converge to a reasonable result starting from an isotropic model with a linearly increasing $v_{n}$, even in absence of low frequencies.

\section{Introduction}

For most seismic data, near and far offsets can't be reproduced simultaneously without accounting for anisotropy. Full waveform inversion (FWI) aims to build a high-resolution subsurface model by minimizing the misfits between the simulated and observed data (Virieux and Operto, 2009). As high-performance computing and the acquired seismic data improve, anisotropic FWI has drawn considerable attentions (Cheng et al., 2014; Operto et al., 2015; Wu and Alkhalifah, 2016; Masmoudi and Alkhalifah, 2018). Even in the simple transversely isotropic medium with vertical symmetry axis (VTI) case, it's very challenging to estimate the anisotropic properties from the pressure wavefield. Acoustic VTI FWI tends to stagnate at an unreasonable model because of the cycle skipping and trade-off issues, especially when the starting model can't describe the kinematics of the true model within half a cycle. Thus, FWI heavily depends on the presence of lowfrequency and long-offset data to construct the longwavelength components of the subsurface model (Li et al., 2018). However, in the absence of low frequencies, FWI requires a good initial model within the basin of attraction of the global minimum.
$\mathrm{Xu}$ et al. (2012) developed the reflection waveform inversion (RWI) method to update the background model along the reflection wavepath by matching the reflections generated from migration/demigration to the observed data. The background updates in RWI requires a true amplitude migration that can be used to predict the reflections with enough accuracy (Guo and Alkhalifa, 2017; Li et al., 2017). $\mathrm{Wu}$ and Alkhalifah (2015) developed a new optimization problem to update the background components and perturbations simultaneously by matching both the diving waves and reflections to the observed data.

Here, we design an acoustic VTI RWI algorithm to retrieve the background components of VTI model (focusing on the parameters that matter in this case) using the reflections in the pressure wavefield. The parameterization of the multiparameter VTI inversion is crucial to the inversion as it controls the potential trade-off between the inversion parameters (Gholami et al., 2013, Kazei and Alkhalifah, 2018). Alkhalifah et al. (2001) pointed out that the kinematical information of $\mathrm{P}$-wave data only relies on $v_{n}$ and $\eta$, if $\delta$ does not change laterally. Based on the analysis of the radiation patterns, the parameterization in terms of $v_{n}, \eta$ and $\delta$ has limited trade-off (Alkhalifah and Plessix, 2014). Li et al. (2017) and Pattnaik et al. (2016) also employed the parameterization given by $v_{n}, \eta$ and $\delta$ to represent the VTI model in their inversion algorithm. For VTI FWI, a combination of the horizontal velocity $v_{h}$ and the parameters $\eta$ and $\varepsilon$ can generate the optimal inversion results especially when the diving waves are available (Alkhalifah and Plessix, 2014; Alkhalifah, 2016).

In this abstract, we invert for the background $v_{n}$ and $\eta$ using the RWI method, which provides a starting model for the VTI FWI. We then perform FWI using a parameterization optimal for the FWI. We test this procedure on a portion of a modified VTI Sigsbee 2A model.

\section{Theory}

We use the 2D pseudo-acoustic VTI wave equation presented by Duveneck et al. (2008) to describe the propagation of the wavefield in VTI media. The coupled second-order differential equations are given as:

$$
\begin{aligned}
& \frac{1}{v_{n}^{2}} \frac{\partial^{2} p}{\partial t^{2}}=\frac{1}{\sqrt{1+2 \delta}} \frac{\partial^{2} q}{\partial x^{2}}+\frac{1}{1+2 \delta} \frac{\partial^{2} p}{\partial z^{2}}+f_{z}, \\
& \frac{1}{v_{n}^{2}} \frac{\partial^{2} q}{\partial t^{2}}=(1+2 \eta) \frac{\partial^{2} q}{\partial x^{2}}+\frac{1}{\sqrt{1+2 \delta}} \frac{\partial^{2} p}{\partial z^{2}}+f_{x}
\end{aligned}
$$




\section{RWI in acoustic VTI media}

where $v_{n}$ is the NMO velocity, $\delta$ is a Thomsen parameter, $\eta$ is the anellipticity parameter and the pressure wavefield is a combination of $p$ and $q$, i.e., $\mathrm{u}=1 / 2(\mathrm{p}+\mathrm{q})$.

Based on the analysis of radiation patterns (Alkhalifah and Plessix, 2014), $\delta$ is sensitive to small scattering angles and can be used to match near-offset reflection amplitudes. The parameter $v_{n}$ with an isotropic sensitivity can also serve as the perturbation to generate the reflections required by RWI. The born modeling operator corresponding to the perturbation of $\delta$ and $v_{n}$ can be expressed as:

$$
\begin{gathered}
\frac{1}{v_{n}^{2}} \frac{\partial^{2} p_{d}}{\partial t^{2}}=\frac{1}{\sqrt{1+2 \delta}} \frac{\partial^{2} q_{d}}{\partial x^{2}}+\frac{1}{1+2 \delta} \frac{\partial^{2} p_{d}}{\partial z^{2}}+\frac{2}{v_{n}^{3}} v_{n d} \frac{\partial^{2} p}{\partial t^{2}} \\
-(1+2 \delta)^{-3 / 2} \delta_{d} \frac{\partial^{2} q}{\partial x^{2}}-2(1+2 \delta)^{-2} \delta_{d} \frac{\partial^{2} p}{\partial z^{2}} \\
\frac{1}{v_{n}^{2}} \frac{\partial^{2} q_{d}}{\partial t^{2}}=(1+2 \eta) \frac{\partial^{2} q_{d}}{\partial x^{2}}+\frac{1}{\sqrt{1+2 \delta}} \frac{\partial^{2} p_{d}}{\partial z^{2}}+\frac{2}{v_{n}^{3}} v_{n d} \frac{\partial^{2} q}{\partial t^{2}}, \\
-(1+2 \delta)^{-3 / 2} \delta_{d} \frac{\partial^{2} p}{\partial z^{2}}
\end{gathered}
$$

where the subscript $d$ denotes the perturbation component. The objective function of RWI in acoustic VTI media can be written as:

$$
J\left(\mathbf{m}, \mathbf{m}_{d}\right)=\frac{1}{2}\left\|\mathbf{u}_{d}\left(\mathbf{m}, \mathbf{m}_{d}\right)-\mathbf{d}_{r e f}\right\|^{2},
$$

where $\mathbf{d}_{\text {ref }}$ represents the observed reflections, $\mathbf{u}_{d}=1 / 2\left(\mathbf{p}_{d}+\mathbf{q}_{d}\right)$ is the perturbed wavefield controlled by equation 2 . Then we can derive the gradients of the objective function with respect to the perturbation model $v_{n d}$ and $\delta_{d}$ using the adjoint state method (Plessix and Cao, 2011):

$$
\begin{aligned}
& \frac{\partial J}{\partial v_{n d}}=\frac{2}{v_{n}^{3}} \int d t\left(\lambda_{q} \frac{\partial^{2} q}{\partial t^{2}}+\lambda_{p} \frac{\partial p}{\partial t^{2}}\right) \\
& \frac{\partial J}{\partial \delta_{d}}==-\int d t(1+2 \delta)^{-\frac{3}{2}}\left(\lambda_{q} \frac{\partial^{2} p}{\partial z^{2}}+\lambda_{p} \frac{\partial^{2} q}{\partial x^{2}}\right)+2(1+2 \delta)^{-2} \lambda_{p} \frac{\partial^{2} p}{\partial z^{2}},
\end{aligned}
$$

and the gradients with respect to the background $v_{n}$ and $\eta$ :

$$
\begin{aligned}
& \frac{\partial J}{\partial v_{n}}=\frac{2}{v_{n}^{3}}\left[\int d t\left(\lambda_{q d} \frac{\partial^{2} q}{\partial t^{2}}+\lambda_{p d} \frac{\partial p}{\partial t^{2}}\right)+\int d t\left(\lambda_{q} \frac{\partial^{2} q_{d}}{\partial t^{2}}+\lambda_{p} \frac{\partial p_{d}}{\partial t^{2}}\right)\right] \\
& \frac{\partial J}{\partial \eta}=2\left[\int d t \lambda_{q d} \frac{\partial^{2} q}{\partial x^{2}}+\int d t \lambda_{q} \frac{\partial^{2} q_{d}}{\partial x^{2}}\right]
\end{aligned}
$$

where $\lambda$ refers to the adjoint wavefield computed from the adjoint operator corresponding to equations 1 and 2 .

The inverse problem in VTI media is an ill-posed problem. A carefully chosen strategy or workflow is a crucial to the stability of the inversion. Alkhalifah (2016) presented transmission radiation patterns from a reflector in the pseudo-depth domain for an acoustic VTI model parameterized by $v_{n}, \eta$, and $\delta$, shown here in Figure 1 . We can see that $\delta$ has no influence on the kinematics of the measured reflections. This further explains why we can't retrieve the background $\delta$ using reflections. Besides, background $v_{n}$ and $\eta$ can be constrained by moderate and far offsets, respectively.
Such analyses suggest the following inversion strategy:

- First, we invert for the background $v_{n}$ using the reflections from the perturbation of $v_{n}$ and $\delta$, which are simultaneously optimized in the least square migration.

- Given the inverted background $v_{n}$, we update the background $v_{n}$ and $\eta$ simultaneously by minimizing the faroffset reflection residuals. The reflections are produced by the perturbation of $v_{n}$.

- Lastly, the inverted VTI model is described by a combination of horizontal velocity $v_{h}, \eta$ and $\varepsilon$ using the following relations:

$$
v_{h}=v_{n} \sqrt{1+2 \eta} \quad \varepsilon=(1+2 \delta) \eta+\delta .
$$

Starting from the background model, VTI FWI is employed to improve the resolution. In detail, the parameter $v_{h}$ is first inverted, and then we add the anisotropic parameters $\eta$ and $\varepsilon$ successively.

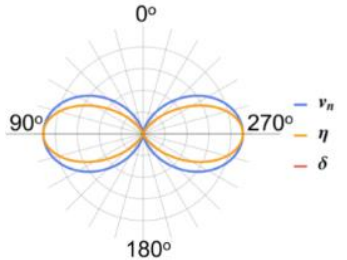

Figure 1: The transmission radiation patterns for scattering from a horizontal reflector in the pseudo-depth domain for an acoustic VTI model. (Courtesy of Alkhalifah, 2016)

\section{Examples}

We test the inversion algorithm using a part of the modified Sigsbee $2 \mathrm{~A}$ model. The true NMO velocity $v_{n}$ is given in Figure 2a. The true $\eta=\delta$ is shown in Figure 2b. The top layer is purely isotropic to avoid the shear wave artifacts caused by the pseudo-acoustic approximation. We use 67 shots to generate the seismic data with the maximum offset of $7.4 \mathrm{~km}$. The inversion result of $\eta$, a parameter that thrives on the large offset information, is influenced by the edge effects because of the limited offset at the edge. Therefore, the model information is limited to mainly the middle part. An 8-Hz Ricker wavelet without frequencies below $3 \mathrm{~Hz}$ is used as the source wavelet. We start from an isotropic model where the NMO velocity is linearly increasing as shown in Figure 3. We first update the perturbation of $v_{n}$ and $\delta$ simutaneously to produce the reflections for the update of the background $v_{n}$. Figure 4 shows the inverted perturbation images using the initial background model, where the reflectors obviously diviate from their accurate spatial position and the diffraction energy are not focused. After thirty ourter iterations, longwavelength components of the NMO velocity $v_{n}$ are recovered well (Figure 5). Correspondingly, the pertubation image is obviously improved (Figure 6). In detail, the reflectors are shifted up to some extent, but still deviate slightly from their exact positions. The depth deviation is mainly caused by the inaccurate $\delta$, which can't be retrieved 


\section{RWI in acoustic VTI media}

from surface seismic data. Besides, the diffaraction energy is not fully focused. According to the above-mentioned inversion strategy, we update the background $v_{n}$ and $\eta$ simultaneously to reduce the reflection residuals at far offsets. During the inversion, the gradient of $\eta$ is considerably smoothed to mitigate possibly tradeoff with the NMO velocity. Figure 7 shows the inverted background $v_{n}$ and $\eta$ after 20 outer iterations. The quality of the perturbation image is improved (Figure 8), especially near the diffraction points. Meanwhile, Figure $7 \mathrm{~b}$ illustrates that the long-wavelength information of $\eta$ is retrieved well except the deep and near the edges, in which they were not illuminated well by the limited aperture reflections. Besides, the accuracy of $\eta$ heavily relies on $v_{n}$.

At last, we conduct acoustic VTI FWI to introduce highwavenumber components. Given the inverted $v_{n}$ and $\eta$ (Figure 7), we re-describe the VTI model with a combination of the horizontal velocity $v_{h}, \eta$ and $\varepsilon$. We first invert for $v_{h}$, and then include the anisotropic parameters $\eta$ and $\varepsilon$ successively. Figure 9 shows the final inversion results. For comparison, we also apply the above FWI algorithm to the isotropic initial model (Figure 3). It fails to get good results, especially for the anisotropic parameters (Figure 10). For a detailed comparison, the vertical profiles at $\mathrm{x}=3.584 \mathrm{~km}$ of $v_{h}, \eta$ and $\varepsilon$ are shown in Figure 11. Due to the tradeoff between the depth and the $\delta$ parameter, the inverted model has a depth deviation. To avoid the tradeoff, we convert the depth axis to vertical time using the inverted parameters, and the corresponding vertical time profiles are shown in Figure 12. The inverted $v_{h}$ is very close to the true horizontal velocity in the vertical time domain. The background components of $\eta$ can also be recovered using the proposed inversion algorithm. This test also points out the ambiguity between $\delta$ and depth which is hard to resolve without additional information. We can see from these Figures that the inverted VTI model interprets the kinematics of the reflections successfully.

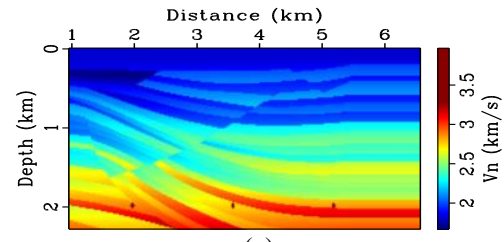

(a)

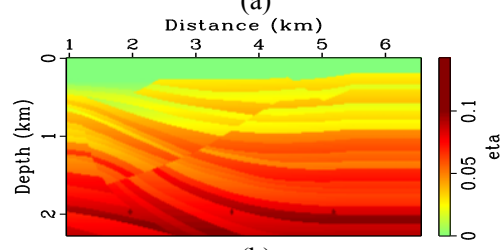

(b)

Figure 2: The true NMO velocity $v_{n}$ and $\eta=\delta$.

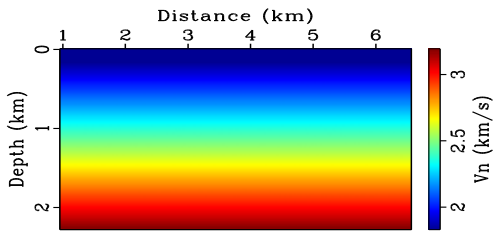

Figure 3: The initial NMO velocity $v_{n}$.

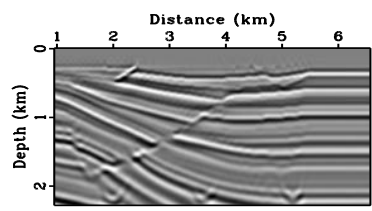

(a)

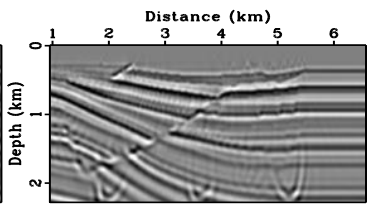

(b)
Figure 4: The perturbation images for $\delta_{d}$ (a) and $v_{n d}$ (b) using the initial model.

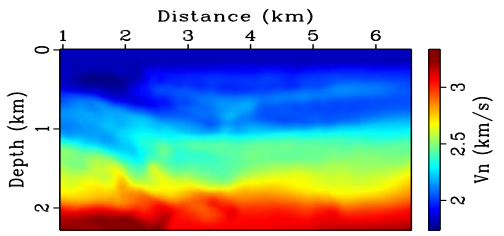

Figure 5: The inverted background $v_{n}$ in the first step of VTI RWI method.

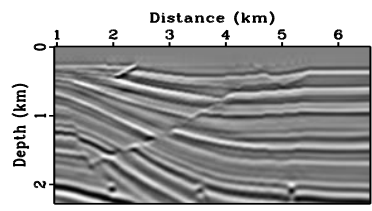

(a)

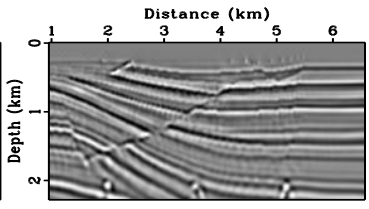

(b)
Figure 6: The perturbation images for $\delta_{d}$ (a) and $v_{n d}$ (b) using the inverted $v_{n}$ (Figure 5).

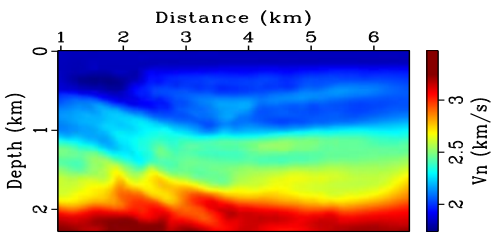

(a)

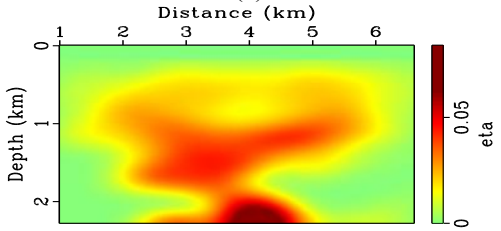

(b)

Figure 7: The inverted background $v_{n}$ (a) and $\eta$ (b) in the second step of VTI RWI method. 


\section{RWI in acoustic VTI media}

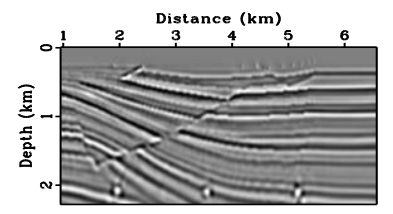

Figure 8: The perturbation images for $v_{n d}$ using the inverted model shown in Figure 7.

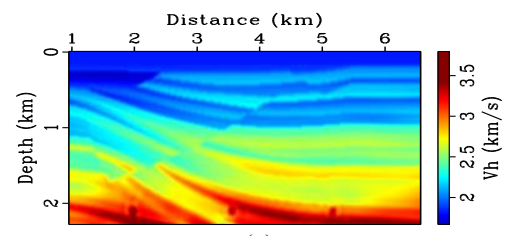

(a)

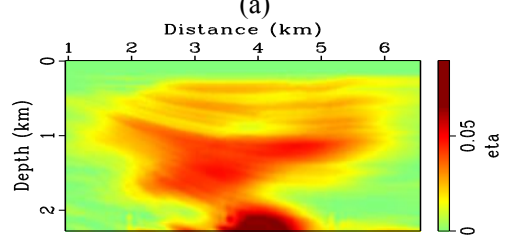

(b)

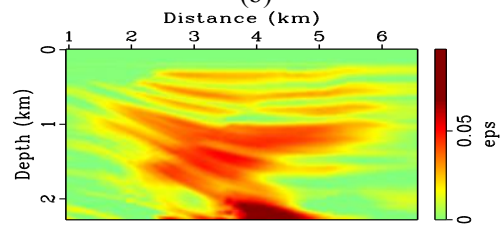

(c)

Figure 9: The VTI FWI results starting from the RWI background model (Figure 7): the inverted $v_{h}(\mathrm{a}), \eta$ (b) and $\varepsilon$ (c).

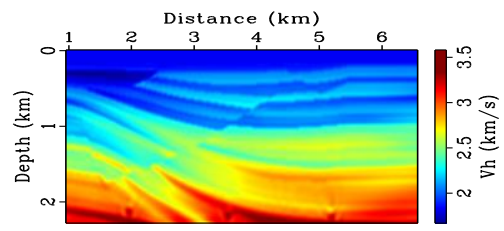

(a)

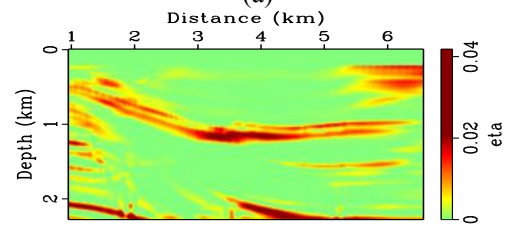

(b)

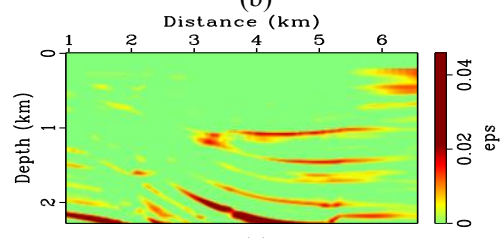

(c)

Figure 10: The VTI FWI results starting from the initial isotropic model (Figure 3): the inverted $v_{h}(\mathrm{a}), \eta(\mathrm{b})$ and $\varepsilon(\mathrm{c})$.

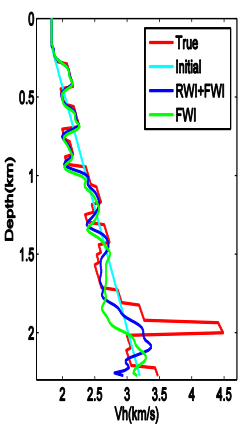

(a)

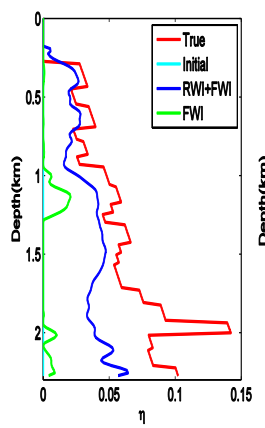

(b)

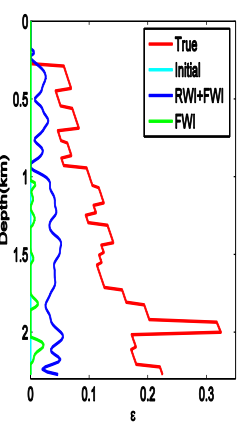

(c)
Figure 11: The vertical profiles at $\mathrm{x}=3.584 \mathrm{~km}$ of $\mathrm{v}_{\mathrm{h}}(\mathrm{a}), \eta(\mathrm{b})$ and $\varepsilon$ (c). (Cyan line: initial model, green line: conventional VTI FWI result, blue line: inverted model and red line: true model)

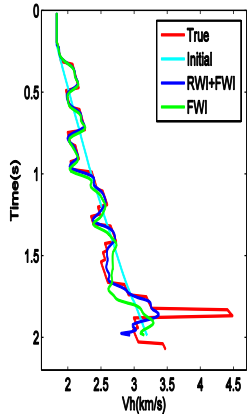

(a)

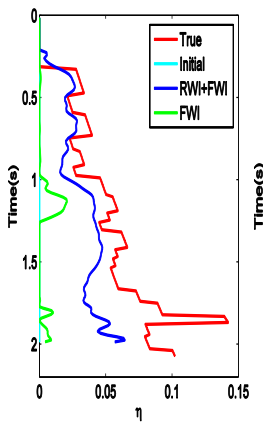

(b)

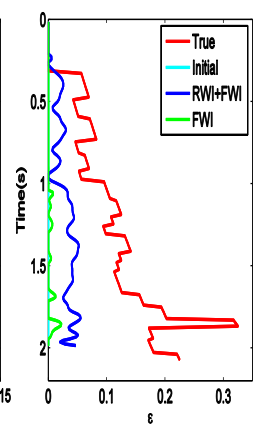

(c)
Figure 12: The vertical time profiles at $\mathrm{x}=3.584 \mathrm{~km}$ of $v_{h}(\mathrm{a}), \eta(\mathrm{b})$ and $\varepsilon(\mathrm{c})$.

\section{Conclusions}

We designed an RWI algorithm to build the background NMO velocity $\left(v_{n}\right)$ and $\eta$ parameters in acoustic VTI media with limited trade-off. We first invert for the background $v_{n}$ by matching the near-offset reflections from the perturbation of $v_{n}$ and $\delta$. Then we optimize the background $v_{n}$ and $\eta$ simultaneously using the reflections generated from the perturbation of $v_{n}$. The numerical test illustrates that the inversion algorithm can recover the background VTI model that interprets the kinematics of the reflections successfully. The sequential VTI FWI result, especially the horizontal velocity $\left(v_{h}\right)$, is very close to the true model in the vertical time domain. In the meeting, we will present an application of the approach on the Volve ocean bottom cable data.

\section{Acknowledgements}

We would like to thank the Shaheen supercomputing Laboratory in KAUST for their computational support. We thank KAUST for its support and SWAG for collaborative environment. 


\section{REFERENCES}

Alkhalifah, T., 2016, Research Note: The sensitivity of surface seismic P-wave data in transversely isotropic media to reflector depth: Geophysical Prospecting, 64, 505-513, doi: https://doi.org/10.1111/1365-2478.12483.

Alkhalifah, T., S. Fomel, and B. Biondi, 2001, The space-time domain: theory and modelling for anisotropic media: Geophysical Journal International, 144, 105-113, doi: https://doi.org/10.1046/j.1365-246x.2001.00300.x.

Alkhalifah, T., and R.-E. Plessix, 2014, A recipe for practical full-waveform inversion in anisotropic media: An analytical parameter resolution study: Geophysics, 79, no. 3, R91-R101, doi: https://doi.org/10.1190/geo2013-0366.1.

Cheng, X., K. Jiao, D. Sun, and D. Vigh, 2014, Anisotropic parameter estimation with full-waveform inversion of surface seismic data: 84th Annual International Meeting, SEG, Expanded Abstracts, 1072-1077, doi: https://doi.org/10.1190/segam2014-0821.1.

Duveneck, E., P. Milcik, P. Bakker, and C. Perkins, 2008, Acoustic VTI wave equations and their application for anisotropic reverse-time migration: 78th Annual International Meeting, SEG, Expanded Abstracts, 2186-2190, doi: https://doi.org/10.1190/1.3059320.

Gholami, Y., R. Brossier, S. Operto, A. Ribodetti, and J. Virieux, 2013, Which parameterization is suitable for acoustic vertical transverse isotropic media full waveform inversion? Part 1: Sensitivity and trade-off analysis: Geophysics, 78, no. 2, R81-R105, doi: https://doi.org/10.1190/geo20120204.1.

Guo, Q., and T. Alkhalifah, 2017, Elastic reflection-based waveform inversion with a nonlinear approach: Geophysics, 82, no. 6, R309-R321, doi: https://doi.org/10.1190/geo2016-0407.1.

Kazei, V., and T. Alkhalifah, 2018, Waveform inversion for orthorhombic anisotropy with P-waves feasibility and resolution: Geophysical Journal International, 213, 963-982, doi: https://doi.org/10.1093/gii/ggy034.

Li, V., H. Wang, I. Tsvankin, E. Díaz, and T. Alkhalifah, 2017, Inversion gradients for acoustic VTI wavefield tomography: Geophysics, 82, no. 4, WA55-WA65, doi: https://doi.org/10.1190/geo2016-0624.1.

Li, Y., Y. Choi, T. Alkhalifah, Z. Li, and K. Zhang, 2018, Full-waveform inversion using a nonlinearly smoothed wavefield: Geophysics, 83, no. 2, R117-R127, doi: https://doi.org/10.1190/geo2017-0312.1.

Li, Y., Z. Li, T. Alkhalifah, and Q. Guo, 2017, Elastic reflection waveform inversion with variable density: 87th Annual International Meeting, SEG, Expanded Abstracts, 1545-1550, doi: https://doi.org/10.1190/segam2017-17723202.1.

Masmoudi, N., and T. Alkhalifah, 2018, Full-waveform inversion in acoustic orthorhombic media and application to a North Sea data set: Geophysics, 83, no. 5, C179-C193, doi: https://doi.org/10.1190/geo2017-0738.1.

Operto, S., A. Miniussi, R. Brossier, L. Combe, L. Metivier, V. Monteiller, A. Ribodetti, and J. Virieux, 2015, Efficient 3-d frequency-domain monoparameter full-waveform inversion of ocean-bottom cable data: Application to Valhall in the visco-acoustic vertical transverse isotropic approximation: Geophysical Journal International, 202, 1362-1391, doi: https://doi.org/10.1093/gji/ggv226.

Pattnaik, S., I. Tsvankin, H. Wang, and T. Alkhalifah, 2016, Full-waveform inversion with reflected waves for 2D VTI media: 86th Annual International Meeting, SEG, Expanded Abstracts, 413-418, doi: https://doi.org/10.1190/segam2016-13967833.1.

Plessix, R.-E., and Q. Cao, 2011, A parametrization study for surface seismic full waveform inversion in an acoustic vertical transversely isotropic medium: Geophysical Journal International, 185, 539-556, doi: https://doi.org/10.1111/j.1365-246X.2011.04957.x.

Virieux, J., and S. Operto, 2009, An overview of full-waveform inversion in exploration geophysics: Geophysics, 74, no. 6, WCC1-WCC26, doi: https://doi.org/10.1190/1.3238367.

$\mathrm{Wu}, \mathrm{Z}$., and T. Alkhalifah, 2015, Simultaneous inversion of the background velocity and the perturbation in full- waveform inversion: Geophysics, $\mathbf{8 0}$, no. 6, R317-R329, doi: https://doi.org/10.1190/geo2014-0365.1.

$\mathrm{Wu}, \mathrm{Z}$., and T. Alkhalifah, 2016, Waveform inversion for acoustic VTI media in frequency domain: 86th Annual International Meeting, SEG, Expanded Abstracts, 1184-1189, doi: https://doi.org/10.1190/segam2016-13867221.1.

Xu, S., D. Wang, F. Chen, G. Lambare, and Y. Zhang, 2012, Inversion on reflected seismic wave: 82th Annual International Meeting, SEG, Expanded Abstracts, 1-7, doi: https://doi.org/10.1190/segam2012-1473.1. 